\title{
LA.UR- $91-2589$
}

Pronind in . ont

DE91 017820

\section{Si.r. 0 O 1991}

Los Alamos Nalional Laboralory is operated by the Universily ol California for Ine Uniled Stales Department of Energy under Coniraci W i G0S. ENG.36

\section{TITLE SPONTANEOUS EMISSION FROM FREE ELECTRON LASERS}

AUthof(S) Mark Jude Schmitl, X-1

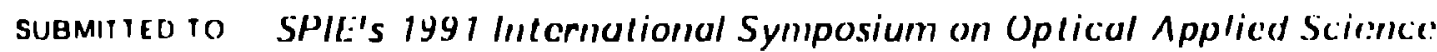
and Engincering.

Short Wavelength Radiation Sources Conference! I.j52,

Sull Diego, (iA,

July $21 \cdot 2 !, 1991$.

\section{ISIS'IAAIMKR}

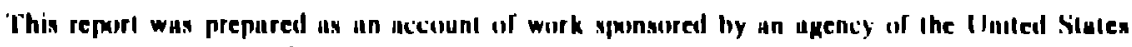
(iovernmeut Neither the Imiled Stules Giuvernment nur uny apency thereur, nur uny of their

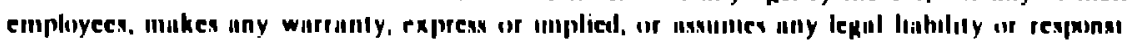

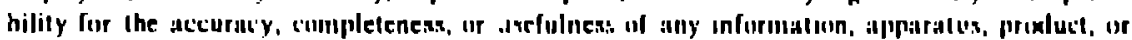

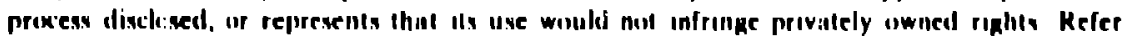

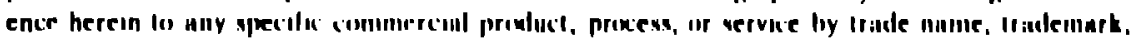

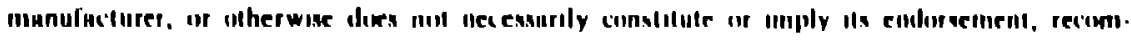

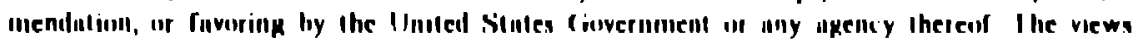

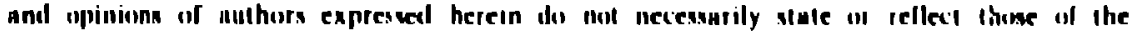
IInitel S'tuten Ciuverninent or any nacncy theress 


\title{
Spontaneous emission from free electron lasers
}

Mark J. Schmill

Los Alamos National Laboratory

MS F645, Los Alamos, New Mexico 87545 USA

\begin{abstract}
Characteristics of the fundamental and harmonic emission from free-electron lasers (FELE) is examilled in the spontarjeous emission regime. The radiation at both odd and evtu harmonic frequencies is treated for electron beams with finite emittance and energy spread. For wigglers with many wiggle periods, calculation of the SE by integrating an ensemble of electrons along their exact trajectories becomes exceedingly cumbersome. Therefore, a different technique is used in which the far-field radiation pattern of a single election is manipulated in transform space to include the effects of emittance. The effects of energy spread can be included by $\varepsilon$ weighted sum over the energy distribution. The program execution time for wigglers of arbitrary length is negigible. The transverse radiation patterns including the transverse frequency dependences, are given. How this radiation is modeled in FEL simulation codes is discussed.
\end{abstract}

\section{INTRODUCTION}

The interest it harmonic radiation from frec electron lasers (FELa) has increased in past few years for several important reasons. The level of apontaneous and coherent-spontaseous harmonic powir generated during fundalliell. tal lasing is of interest from both a mirror damage standpoint and in ternu of the use of this radiation for diagnostics and external experimentation. Alheit broadloand in frequency, spontaneous eminsion is the dominant radiation merhanism at the higher harmonics. This radiation diverges faster than the coherent-spontaneous radiation and ian sulfer sugnificant interc ?ption ard reflection from the wiggler vacuum tube wall. Its use as an alignment diagnostie call also be important. The prediction of the spont aneous emission power levele for FEL in the nubjert of this paper.

In Sectior. 2 the mathematical nodels for the epontaneous emission fron a single electron and from an entire electron beam (including emittance effects) are given. (Computational examples using these models follesws. llow spontaneous emission is modeled in the present PEL codes is discusased in Section 3. A breif dessription of ione thase models can be used to ealculnte the spontaneous emisaion from electromagnetic wigglers is given in Section 1 . 'Tlue conclusions are given in Section 5 .

\section{ANALYUICAL MODLL}

Filectrons eadiate spontancously in a wiggler. Mest of the tadiation is contained in a forward directed cour. of lialf angle $a_{u} / \gamma$. The far fiell radiation intensity for a ningle clectron was annlyticnlly derive i by ('olecon'. Jul his derivation lie correctly included all termen

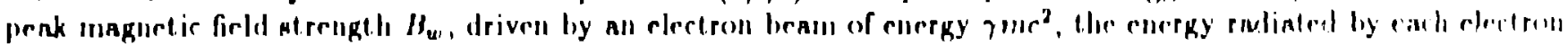
of charge $q=-r$ per unit molid angle and frequerucy bnodwidth is given by

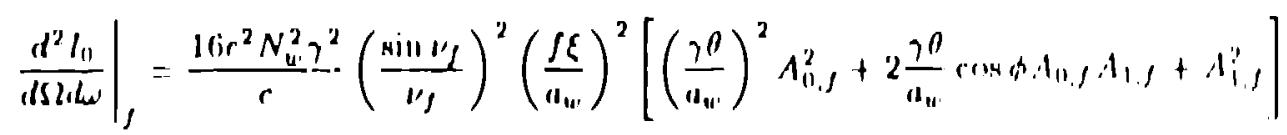

where

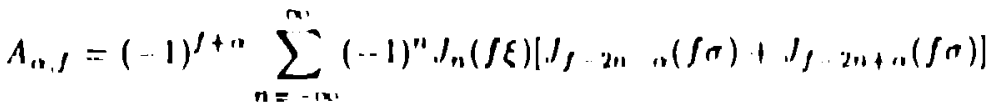

$$
\begin{aligned}
& { }^{\prime \prime} \rho=\pi N_{w}\left[\rho \cdot \frac{\lambda_{u}}{27^{2} \lambda_{0}}\left(1+a_{u}^{2} / 21 \gamma^{2} 0^{2}\right)\right]
\end{aligned}
$$




$$
\begin{gathered}
f \sigma=\frac{8(f \xi)(\gamma \theta)}{a_{w}} \cos \phi \\
\xi=\frac{a_{w}^{2}}{4\left(1+a_{w}^{2} / 2+\gamma^{2} \theta^{2}\right)}
\end{gathered}
$$

and

$$
a_{w}=\frac{|c| B_{w} \lambda_{w}}{2 \pi m c^{2}}
$$

is the normalized wiggler magnetic vector potentıal. The radiation wavelength is $\lambda_{1}, f$ is the harmonic number and $J_{m}(z)$ represents the Bessel function of the first kind of order $m$. As depicted in Figure 1 , it has been asaumed that the wiggler lies along the z-axis and the observation angles $\theta$ and $\phi$ are formed by the intersection of the line of observation, call it $\hat{n}$, with the $z$-axis and the angle made by the intersection of the projection of $\dot{n}$ onto the $x y$-plane with the $\boldsymbol{x}$-axis, respectively. It should be noted that other derivations, $\mathbf{2}^{\mathbf{3}}$ omil an axial phase term, therehy oblaining an erroneous azimuthally aymmetric result. The harmonic radiation paltern described in Eq.(1) has multip!r lobes where the number of lobes is equal to the harmonic number. $A$ cold electron beam (zero energy spread and emitt ance) has a radiation pattern that is identical in shepe to the single-electron pattern.

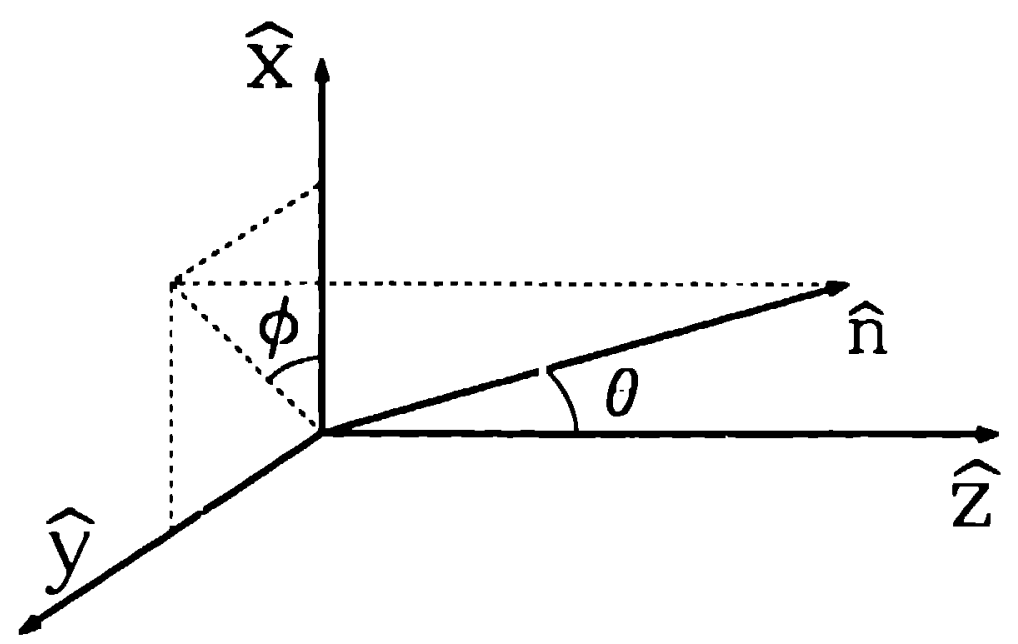

Figure 1. Heintion between the cartenian coordinates, the lite of obervation and the splierical observation anglen.

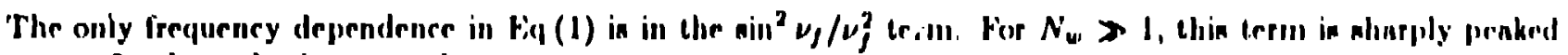
about $\nu_{f}=0$, where the irequeney berounen

$$
w=\frac{2 \gamma^{2} \int k_{u}, r}{1+a_{w}^{2} / 2+\gamma^{2} g^{2}}
$$

An on-axin ohererver $(\theta \Rightarrow(!)$ aren thir frequirury

$$
w_{\text {an1: }}=\frac{2 \gamma^{2} k_{u \cdot r f}}{1+a_{u}^{2} / 2}
$$

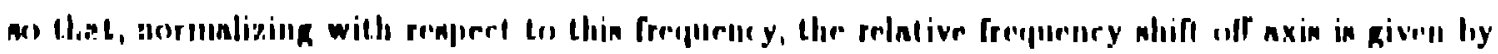

$$
\frac{\omega}{w_{n+10}}=\frac{1}{11\left(\frac{x^{2}{ }^{2} 1}{1+a^{2} 12}\right)}
$$




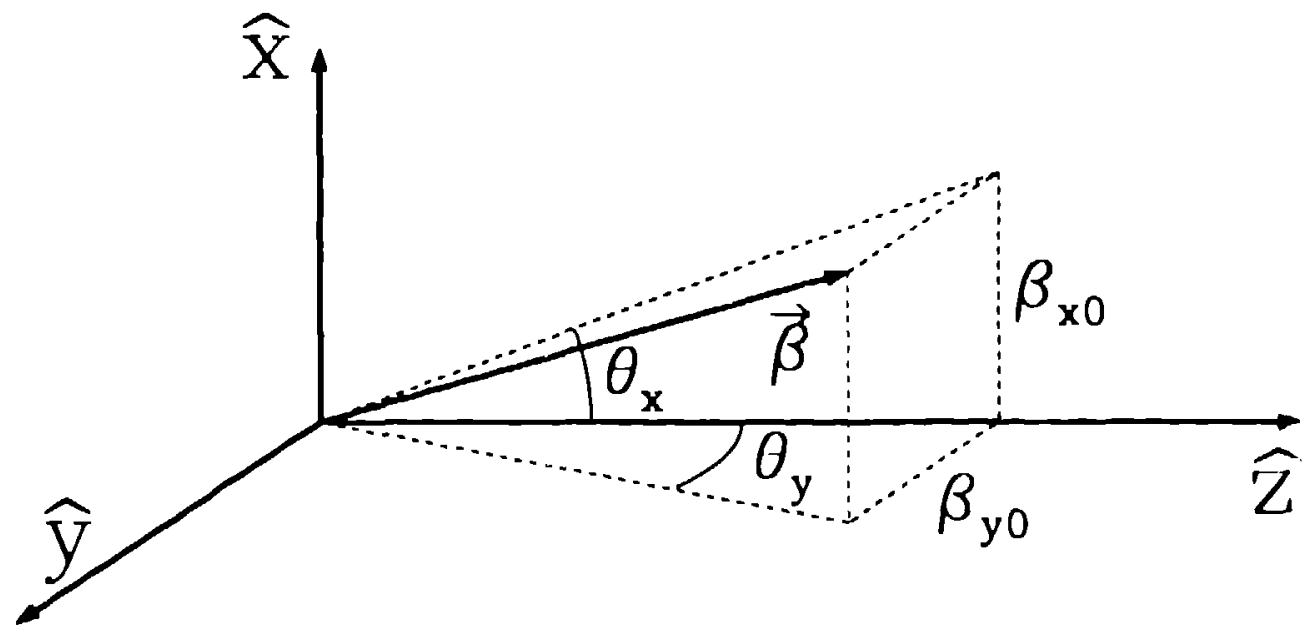

Figure 2. Relation between the electron guiding center velocity, $\vec{\beta}$, its transverse drift speeds and the two angles of rotation.

From Eq.(9) one sees that at the edge of the $\gamma \theta \simeq 1$ cone, the frequency has changed by :oughly a factor of two. It should also be noted that since the frequency depends only on $\theta$ (and not $\phi$ ), a specific frequency appears spatially as a "frequency ring".

Spontaneous emission from an FEL is composed of the radiation from all the electrons in the electron beam. Since the electron beam has a distribution of electrons in enetgy, opace and transverse velocity, sorie method must be devised to take these distributions into arcount in order to obtain the correct spontancous emission pattern for a non-ideal electron beam. As a first step we can show that the apontaneous emission of an clectron with a transverse drift (with respect to the wiggler axis) is identical (lo order $1 / \gamma^{2}$ ) in both intensity and frequency to the spontaneous radiation pattern of the on-bxis electron. The only difference is that the drifting electron's radiation paltern is rolated by $\vec{\beta}_{s 0}+\vec{\beta}_{y 0}$ away froin the wiggler axis where $\vec{\beta}_{z 0}$ and $\vec{\beta}_{y 0}$ correapond to the drift velocitien in the $\dot{x}$ and $\dot{y}$ directions, respectively. The velocity of an electron in a sinumoidal wiggler field including iranaverse drift motion is given by

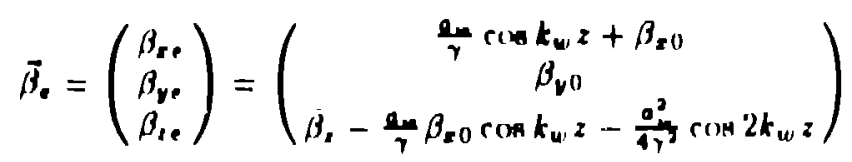

where

$$
\dot{\beta}_{\alpha}=1-\frac{1+a_{u}^{2} / 2}{2 \gamma^{2}}-\beta_{10}^{2} / 2
$$

and

$$
\beta_{\perp 0}^{2}=\beta_{r 0}^{2}+\beta_{v 0}^{2}
$$

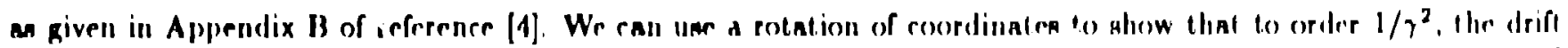

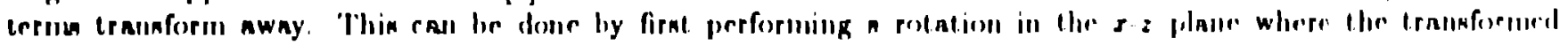
velocitien (denoted by 1) nere given hy

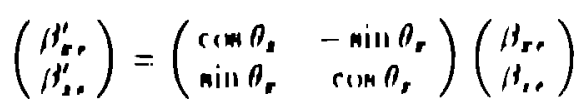

followed by a rotention in the $y$ z plane with the trantionution

$$
\left(\begin{array}{l}
A_{y^{\circ}}^{\prime} \\
H_{y}^{\prime}
\end{array}\right)=\left(\begin{array}{cc}
\cos \theta_{y} & -\sin \theta_{y} \\
\sin \theta_{y} & \cos \theta_{y}
\end{array}\right)\left(\begin{array}{l}
H_{y^{e}} \\
H_{1 .}
\end{array}\right)
$$

where Or alid t, are dellined in rigure? 
Combining the two transformations into one $e^{b}$, the complete transformation can now be written

$$
\begin{aligned}
\left(\begin{array}{c}
\beta_{x e}^{\prime} \\
\beta_{y e}^{\prime} \\
\beta_{x e}^{\prime}
\end{array}\right) & =\left(\begin{array}{ccc}
\cos \theta_{x} & 0 & -\sin \theta_{x} \\
0 & 1 & 0 \\
\sin \theta_{x} & 0 & \cos \theta_{x}
\end{array}\right)\left(\begin{array}{ccc}
1 & 0 & 0 \\
0 & \cos \theta_{y} & -\sin \theta_{y} \\
0 & \sin \theta_{y} & \cos \theta_{y}
\end{array}\right)\left(\begin{array}{c}
\beta_{x e} \\
\beta_{y e} \\
\beta_{x e}
\end{array}\right) \\
& =\left(\begin{array}{ccc}
\cos \theta_{x} & -\sin \theta_{x} \sin \theta_{y} & -\sin \theta_{x} \cos \theta_{y} \\
0 & \cos \theta_{y} & -\sin \theta_{y} \\
\sin \theta_{x} & \cos \theta_{x} \sin \theta_{y} & \cos \theta_{x} \cos \theta_{y}
\end{array}\right)\left(\begin{array}{c}
\beta_{x e} \\
\beta_{y e} \\
\beta_{z e}
\end{array}\right)
\end{aligned}
$$

Now, since the electron's velocity is mainly in the $\dot{z}$-direction, we can approximate

$$
\sin \theta_{x, y} \simeq \beta_{x 0, \nu 0} / \beta, \simeq \beta_{x, y 0}
$$

and

$$
\cos \theta_{x, y} \simeq 1-\theta_{x, y}^{2} / 2 \simeq 1-\beta_{x 0, y 0}^{2} / 2
$$

and denoting $\beta_{x}=\left(a_{w} / \gamma\right) \cos k_{t} z$ and

$$
\beta_{z}=1-\frac{1+a_{u}^{2} / 2}{2 \gamma^{2}}-\frac{a_{u}^{2}}{4 \gamma^{2}} \cos 2 k_{w} z
$$

the transformation equation becomes

$$
\left(\begin{array}{c}
\beta_{x e}^{\prime} \\
\beta_{y e}^{\prime} \\
\beta_{s e}^{\prime}
\end{array}\right)=\left(\begin{array}{ccc}
1-\beta_{x 0}^{2} / 2 & -\beta_{x 0} \beta_{y 0} & -\beta_{x 0} \\
0 & 1-\beta_{y 0}^{2} / 2 & -\beta_{y^{0}} \\
\beta_{x 0} & \beta_{y 0} & 1-\beta_{\perp 0}^{2} / 2
\end{array}\right)\left(\begin{array}{c}
\beta_{x}+\beta_{x 0} \\
\beta_{y 0} \\
\beta_{L}-\beta_{x 0} \beta_{x}-\beta_{\perp 0}^{2} / 2
\end{array}\right)=\left(\begin{array}{c}
\beta_{x} \\
0 \\
\beta_{u}
\end{array}\right)
$$

where we have kept only terms up to order $\mathcal{O}\left(1 / \gamma^{2}\right)$ and assumed $\beta_{r 0, y 0} \times 1 / \gamma$. As seen from Fq.(19), the drift terms have been transformed away leaving expressions identical to thoac for an electron on axis. Thrrefore, the radiation and frequency paltern of a drifting electron is identical to an on-axis electron, with only a shift of the palterll hy $\vec{\beta}_{10}$. (An additional assumption $\lambda_{w} \simeq \lambda_{w}\left(1+\beta_{\perp 0}^{2} / 2\right)$ has also been made.)

With the previous result in mind, we can postulate that the radiation patern fiom an ensetuble of rlectrons is given by

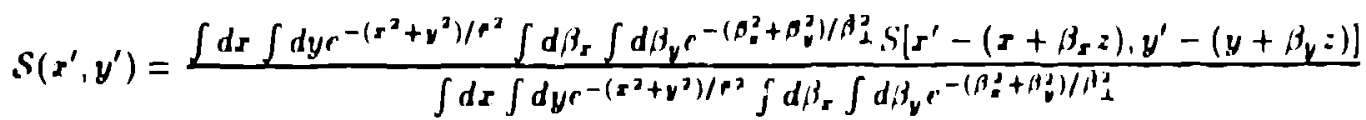

where $S$ in the aingle-electron radiation pallern and $z$ is the axial observalion pount. In Fiq.(20), gaussian diatributions

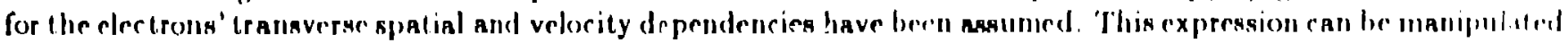
into the form

$$
S\left(s^{\prime}, y^{\prime}\right)=\mathcal{F}^{-1}\left\{c^{-2 B\left(r^{2}+A_{1}^{2},^{2}\right)\left(k^{2}+k^{2}\right)} \dot{S}^{(}\left(k_{s}, k_{v}\right)\right\}
$$

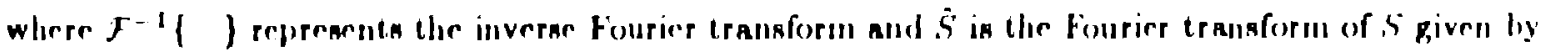

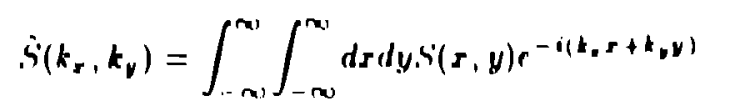

In the fHI field we rall aMHIIIII

$$
s=\left.\frac{d l}{d S l l^{\prime}}\right|_{1}
$$

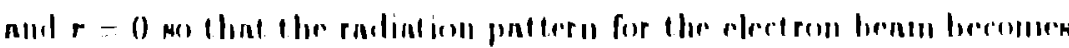

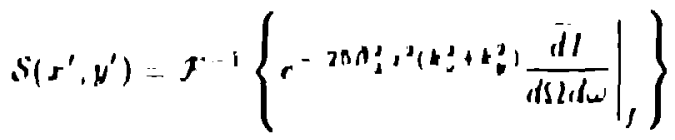


where again, the tilde represents the transformed quantity. Using Eqs.(1)-(6) and (24) the spontaneous emission for a single electron or an entire electron beam with a given energy spread and emittance can be obtained.

The previously described spontaneous emission model has been incorporated into the code SPEMIT. A finite oummation is performed prosiding an excellent approximation for $\mathrm{Eq}(2)$. Afler $\mathrm{Eq}(1)$ has been calculated on an $x$-y grid, an FFT routine is used to obtain its Fourier transform. Multiplication by the angular divergence factor is then performed, after which the FFT routine is used to perform the inverse transform yielding the radiation pattern for the electron ensemble. An example of the code output is given in Figure 3. Herc the far-field single-electron radiation pattern at the sixth harmonic is depicted for the 5.0 meter wiggl.r on the Argonne Advanced Photon Source (APS). Other parameters used for the simulation were: $\lambda_{w}=3.3 \mathrm{~cm}, \gamma=13700 ., B_{w}=3788.0 \mathrm{Gauss}$, and $I=0.1 \mathrm{Amps}$. The frequency dependence, as given in Eq.(7), is shown as color variation over the plot. Note that the number of maxima is equal to the harmonic number.

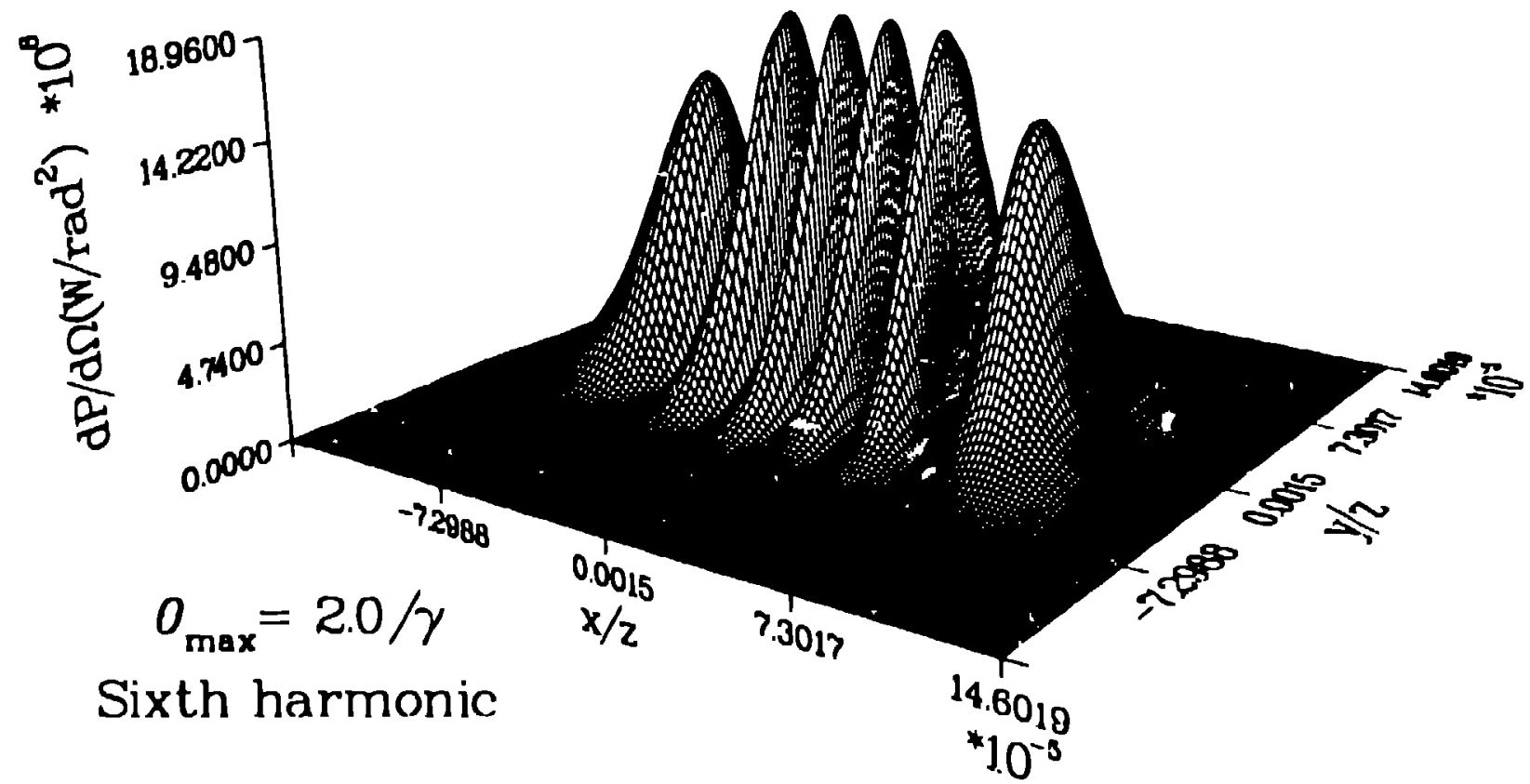

Figure 3. Plot of the ningle-plectron spontaneous emimglun pattern at the sixth harmonic for the Argonlur Als wiggler. Frequeney elinuges are depicted ill color.

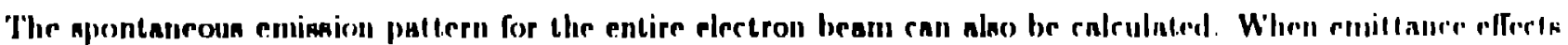

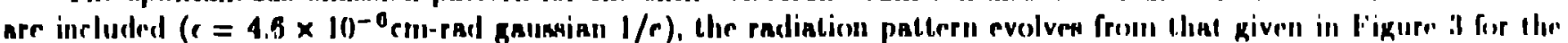

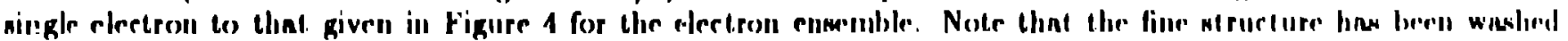

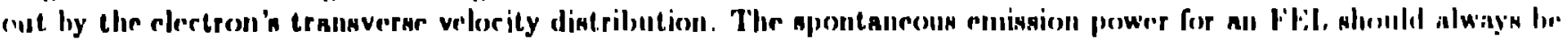

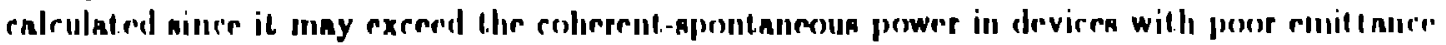

\section{SIMULAUUN OE NOLSEIN FELI' AND FELEX}

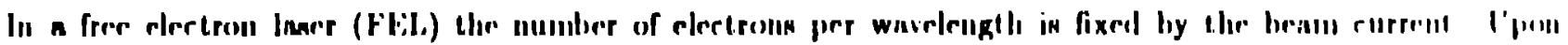

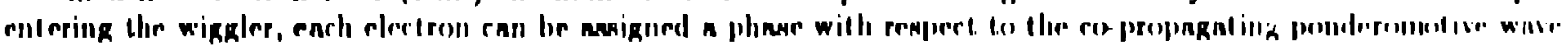




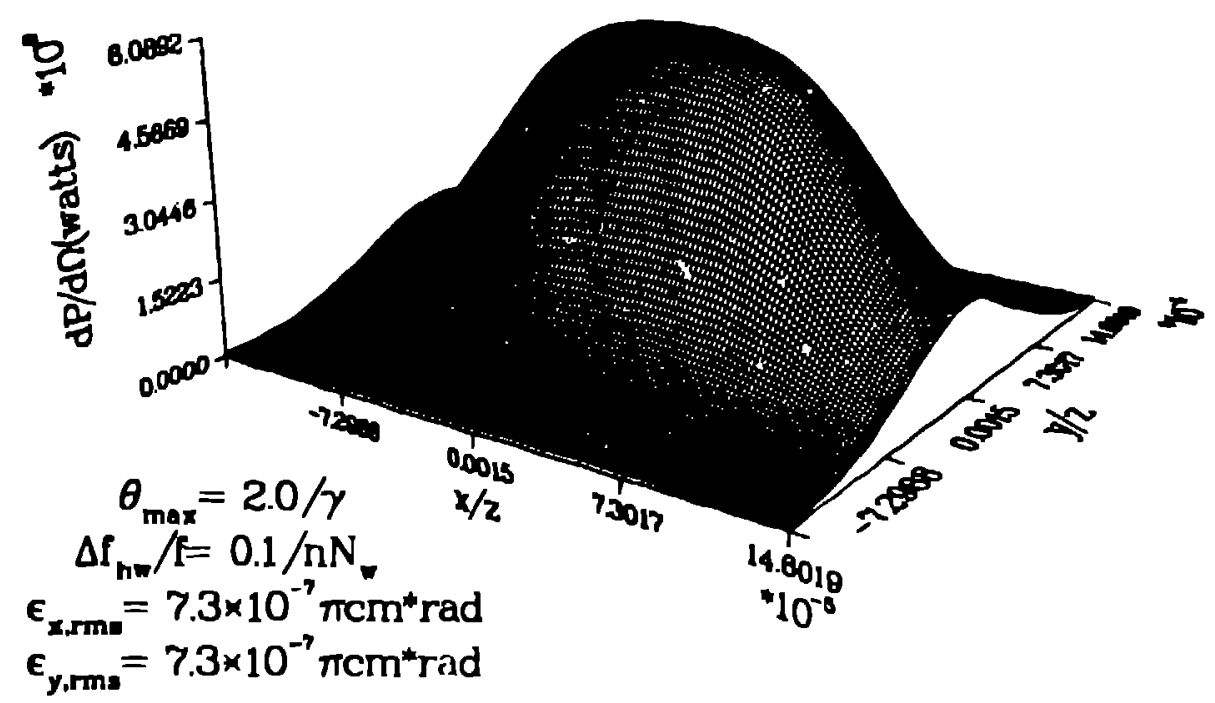

Figure 4. Spatial plot of the sixth harmonir spontaneous enission intensity for the Argonne APS wiggler. 'The distribution of trangverse velocities due to emittance has smeared the single-elertron pattern.

traveling at alightly less than the electron's axial apeed. Variations in the electron current can thrrefore. i, mapprd into variations in the axial electron density in phase space. This variation gives rise to shot noiss. This noise is different from quantum noige which arises due to the randon temporal enission of photons by each elect ron inside. the wiggler. This analysis does not include any quantum noige effects which others have shown ${ }^{6}$ to be negligible in our regime of interest.

For our purposes we can assune the ponderomotive wavelength is equal to the optical field wavelength (high-y approxionation). 'The contribution from each electron to the optical wave can be nodeled by a conplex phasor given by $^{7}$

$$
t_{i}=a_{i} r^{i \phi}
$$

where $\alpha_{1}$ is the amplitude of the phasor representing the $i^{\text {th }}$ electron $\left(\alpha_{i} \propto q_{1} / \gamma_{1}\right)$ and $\phi_{1}$ is its phase. Denorting the number of electruns per optical wavelengih as $N_{e}$, lhe resultant phasker is given by

$$
t=\sum_{i=1}^{N_{1}} n_{1} r^{i \phi_{1}}
$$

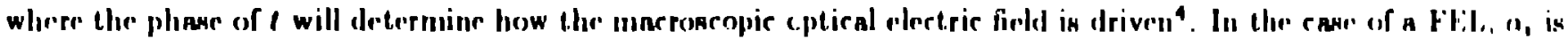

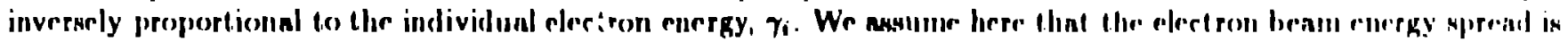
nimnll, $\Delta \gamma / \gamma \ll$ I murh that all rlect.rons huve equal amplitude.

'The ranl and imaginary' part.e of 1 are just.

$$
\left[\begin{array}{l}
r \\
i
\end{array}\right]=\sum_{i=1}^{N_{g}} a_{1}\left[\begin{array}{l}
\operatorname{com} \phi_{1} \\
\sin \phi_{1}
\end{array}\right]
$$

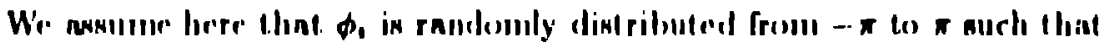

$$
\overline{\operatorname{rim} \phi_{1}}=\overline{H_{1} \| \phi_{1}}=0
$$


where the bar denotes the average value of $\cos \phi_{i}$. It follows trivially that

$$
\left[\begin{array}{l}
\bar{r} \\
\bar{i}
\end{array}\right]=\sum_{i=1}^{N_{i}} \overline{\alpha_{i}}\left[\overline{\frac{\cos \phi_{i}}{\sin \phi_{i}}}\right]=0
$$

where the averaging process should not be confused with the sum over $i$ and we have used the fact that $\bar{\alpha}_{i}=\bar{\alpha}_{n}=\bar{\alpha}_{\text {. }}$ Evaluating the second moments we have

$$
\begin{aligned}
& \overline{r^{2}}=\sum_{i=1}^{N_{1}} \sum_{j=1}^{N_{6}} \alpha_{i} \alpha_{j} \cos \phi_{i} \cos \phi_{j} \\
& \overline{i^{2}}=\sum_{i=1}^{N_{1}} \sum_{j=1}^{N_{6}} \alpha_{i} \alpha_{j} \sin \phi_{i} \sin \phi_{j}
\end{aligned}
$$

and since the amplitudes and phases are all independent of one another we can write

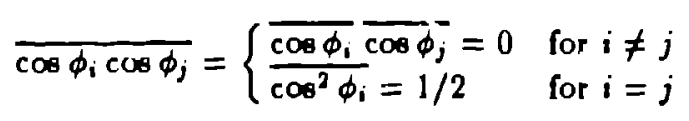

and similarly for the sin terms, such that

$$
\begin{aligned}
\overline{r^{2}}=\overline{i^{2}} & =\sum_{i=1}^{N_{e}} \frac{\overline{\alpha_{i}} \overline{a_{i}}}{2} \\
& =\sum_{i=1}^{N_{e}} \frac{\overline{a^{2}}}{2} .
\end{aligned}
$$

For our case, all vectors (electrons) have essentially equal amplitude $\left(\gamma_{i} \simeq \gamma_{0}, q_{i}=-e\right)$ so that

$$
\overline{r^{2}}=\overline{i^{2}}=\frac{N_{c} \cdot \overline{a^{2}}}{2} .
$$

For any random variable the varianice in given by

$$
\sigma^{2}=\overline{u^{2}}-\ddot{u}^{2}
$$

on that using Ega.(29), (33) and (34) the variance for the resl part (or imagimary part) of $t$ is given by

$$
\sigma_{p}^{2}=\frac{N_{e} \overline{a^{2}}}{2}
$$

where the subacript nignifies that, this in the experinental (or physical) varianer

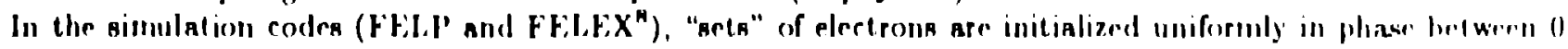
and $2 \pi$ much that, for carli art containing $N_{\text {p electrons }}$

$$
t_{1}=\sum_{i=1}^{N} a r^{i v_{1}}=0
$$

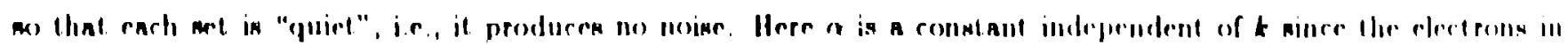

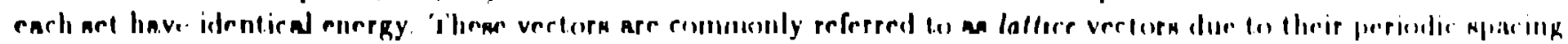

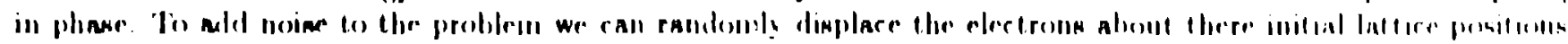


Mathematically, this is accomplished by rotating each lattice vector by a small amount, $\delta \psi_{\mathbf{k}}$, so that, for each set of electrons

$$
t_{0}=\sum_{k=1}^{N_{i}} \alpha e^{i\left(\psi_{k}+\delta \psi_{k}\right)} \neq 0
$$

which can be approximated for small $\delta \psi_{k}$ as

$$
t_{k}=\sum_{k=1}^{N} \alpha e^{i \psi_{k}}\left(1+i \delta \psi_{k}\right)
$$

where $\delta \psi_{k}$ is the initial phase displacement of the kth electron. Assuming $N$, sets of electrons are modeled, the resultant phasor (represeniing all the simulation electrons) is given by

$$
t=\sum_{l=1}^{N_{1}} \sum_{k=1}^{N_{k}} a_{l}\left[e^{i \psi_{k}}\left(1+i \delta \psi_{k}\right)\right]_{l}
$$

where the orientation between $N$, sets in phase is random. Using Eq.(36) and defining $\bar{\psi}_{k}=\psi_{k}+\pi / 2$, this expression reduces to

$$
t=\sum_{l=1}^{N_{l}} \sum_{k=1}^{N_{p}} \alpha_{l}\left[\delta \psi_{k} e^{i \bar{\psi}_{l}}\right]_{l}
$$

and lettirg the sotal number of simulation electrons, $N_{t}=N_{1} N_{p}$, gives

$$
t=\sum_{t=1}^{N_{1}} \alpha_{0} \delta \psi_{i} e^{i \psi_{t}}
$$

where we assumed $\alpha_{l}=\alpha$, is a constant. This equation has the sare form as Eq.(26) for the experimental case Therefore, we can use Eqs.(32) and (34) to obtain

$$
\begin{aligned}
\sigma_{0}^{2} & =N_{1} \overline{a_{0}^{2}} \frac{\overline{\delta \psi^{2}}}{2} \\
& =N_{1} \alpha^{2}\left(\frac{N_{e}}{N_{1}}\right)^{2} \frac{\overline{\delta \psi^{2}}}{2}
\end{aligned}
$$

where $\alpha_{0}$, the annplitude of the simulation electron phasor, has been replaced by the number of experimental elertrons it represente

$$
\alpha_{0}=\alpha\left(\frac{N_{e}}{N_{1}}\right) \propto \frac{-c}{\gamma_{0}}\left(\frac{N_{e}}{N_{1}}\right)
$$

in order to obtain the correct maxroneopic current.

Since both the real FEL and the eystem model contain many electrons, both of their probalsility density functions

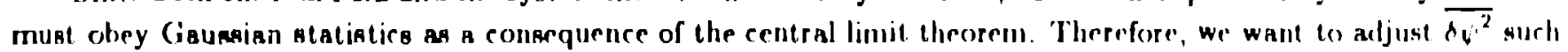
that the name amount of power is radiated for both cases.

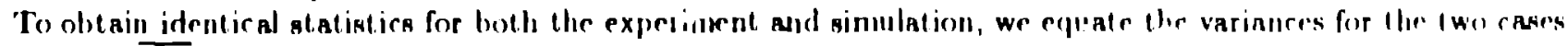

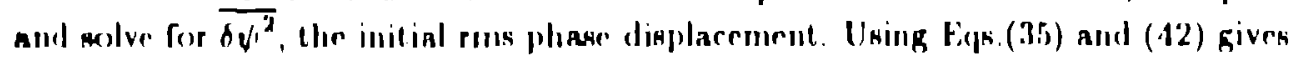

$$
\frac{N_{e}}{2}=N_{1}\left(\frac{N_{e}}{N_{1}}\right)^{2} \frac{\overline{\delta \psi^{2}}}{2}
$$

or

$$
\phi v_{r m}=\sqrt{\phi v^{2}}=\left(\frac{N_{1}}{N_{e}}\right)^{1 / \partial} .
$$


For the harmonics where $N_{e} \rightarrow N_{e} / f, N_{t} \rightarrow N_{t} / f$, and $\psi_{k} \rightarrow f \psi_{k}$ the rme harmonic phase noise is given by

$$
f \delta \psi_{\mathrm{rml}}=\left(\frac{N_{\mathrm{t}}}{f N_{\mathrm{e}}}\right)^{1 / 2} .
$$

Thus, the standard deviation of the simulation electrons from their uniform positions should be much less than the $-\pi$ to $\pi$ variation that exists when all the electrons are simulated. This insures that the simulation electric-field noise in the forward direction will be equivalent to spontaneous emission in the experiment. Note that when simulating the harmonic radiation, $N_{p}$ must be adjusted so thid Eq.(36) holds for the harmonic of interest. This can easily be accomplished by setting $N_{p}$ equal to twice the harmonic number.

For multi-dimensional simulalions, radial noise arizes in the transverse field profile due to the fluctuations in the axial phase average $(t)$ for different transverse locations. The wavelength of the light being simulated determines the minimum structure size of these fluctuations. Thus, the transverse grid spacing should be roughly equal to the optical wavelength for the proper radial averaging to take place. Coarser grid spacing would presumable over estimate this vveraging thereby requiring a longer time for the laser to start up.

A question often arizes regarding the noise observed off-axis from a set of electrons initialized uniformly on axis (quiet). This picture is inconsistent with the way the electric field is cslculated, i.e.

$$
E \propto\left\langle\frac{e^{i \psi}}{\gamma}\right\rangle_{e^{-}}
$$

so that the electric field source at any iransverse location depends only on the arial location of the electrons at that transverse location. Additional off-axis field contributions frcm diffraction are then calculated by the field propagator.

The normalized spectral intensity calculated using Eq.(45) in the one dimensional FEL code FELP is given in Figure 5. This data was generated by averaging the spectral intensities from many passes thereby allowing the simulation to be conducted with relatively few simulation electrons per pass. The results reproduce the $\sin ^{2} x / x^{2}$ behavior exhibited in Eq.(1). Paramelers for the simulation were; $\lambda_{w}=1 \mathrm{~cm}, B_{w}=12000$ Gauss, $\gamma=370$, $I_{\text {peak }}=125 \mathrm{Amps}$. The electron beam was assumed to be cold so that the spectral fringes would be well defined. Using this model, calculation of the spectral lineshapes for tapered wigglers and/or electron beams with einittance and energy spread is possible. Further three dimensional calculations (FELEX) are planned to compare the angular power spectrum with the analytical model.

\section{ELECTROMAGNETLC WJGGLERS}

The models for spontaneous emission described in Sections 2 and 3 assumed wigglers constructed of magnetic malerials. However, theae models can also valid for FEL interactions where the wiggler consists of an electromagnetic wave, provided ome simple modifications are made to the model. An electromagnetic wave colliding with an electrcs beam produces a sinusoidal oscillation of the electrons. In the high gamma limit, this inotion is identical to that induced by a magnetic wiggler with

$$
\lambda_{w}=\lambda_{e m} / 2
$$

where $\lambda_{w}$ is the magnetic wiggler period and $\lambda_{\mathrm{em}}$ is the wavelengll, of the optical wiggler field. The allatogous effective wiggler vector potential is given by

$$
a_{w}=8.6 \times 10^{-6} \sqrt{\frac{P_{w 1 g}(W) \lambda_{e m}}{z_{r}}}
$$

where P'wa in the optical wiggler power and $z_{r}$ is the Rayliegh range of the optical wiggler fie!d in the interaction region. The rquivadent wiggler magnetic firld. $H_{w}$, in given by

$$
H_{u}=\frac{a_{u}}{9.3: 37 \lambda_{u}}
$$




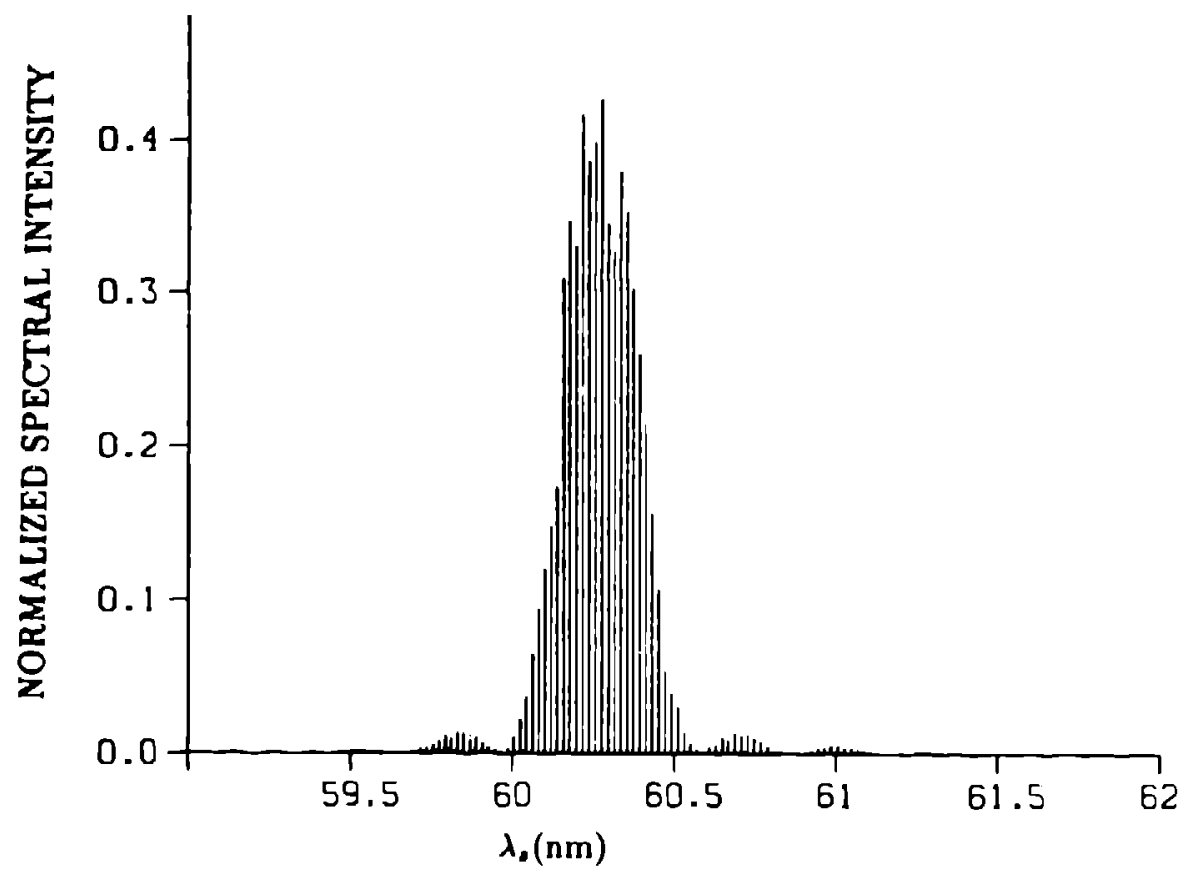

Figure 5. Spectral intensity generated with electron phase jitler in the one dimensional FEL code FELP. The intensity envelope is in excellent agreement with Eq.(1) for $\theta=0$.

Several modifications to the simulation codes are required to properly model electromagnetic wigglers. The cosh transverse dependence of a magnetic wiggler must be replaced with the gaussian dependence of an optical wiggler. The spotsize of the optical wiggler must be judiciously chosen (e.g. some multinle of the electron becin radius). A thorough model would also include the gaussian amplitude and phase depender. I the the optical wiggler field. The focusing effects of the magnetic wiggler must also be removed from the elect. $\therefore$. aquations of motion.

\section{CONCLUSIONS}

The ability to model spontaneous emission from FELs has been demonstrated. The models are three-dimensional in nature and include the effects of energy spread and emiltance. The frequency lineshape of the spontancous emission generated by electron beams with arbitrary emittance and energy spread in wigglers with tapered or unt apered profiles can be obtained by using the electron noise model in the FFL simulation codes. With a few modifications these. codes can also be used to model the spontaneous emission from electromagnetic wigglers. Addition work to model spontaneous emission with the simulation code FELEX is planned.

\section{ACKNOWLEDGMENTS}

'The authror would like to thank John Goldatein and Brian MeVey for useful discussions on the topies covered in this paper.

\section{REEERENCES}

1. W. B. Colson, G. Daltoli and F. Ciocci, "Angular-gain Spectrum of Free-rlectron Lasers", Phys Rev. A. 31. pp. $828-842,(1985)$.

2. B. M. Kincaid, "Random Errora in Undulatora and Their Efferts on the Radiation Spertrum", J. Opl. Sor 
Am. B 2 pp. 1294-1306, 1935.

3. S. Krinsky, “Undulatora as Sources of Synchrotron Radiatius”, IEEE Trans. on Nuclear Science, NS-30, PF. 3078-3082, 1983.

4. M. J. Schmitt and C. J. Elliott, "Generalized Derivation of Free-electron-laser Harmunic Radiation from Planepolarized Wigglers", Phys. Rev. A41, pp. 3853-3866, 1990.

5. H. Goldstein, . Jassical Me hanics, Section 4.4, Addison-Wesley Publishing Company, Reading, Massachusetls, $195 \mathrm{C}$.

6. S. Bensen and J. M. J. Madey, "Shot and Quantum Noire in Free Electron Lasers", Nucl. Instrum. Methods Phys. Res. A 237, J. M. J. Madey and A. Renieri edilors, pp. 55-60, North-Holland, Amsterdam, 1985.

7. This terivation closely foliows that given in Section 2.9 of J. Goodman, Statistical Qutics, Wiley, New York, N York, 1985.

8. B. D. McVey, J. C. Golistein, R. L. Tokar, C. J. Elliott, S. J. Gitoner, M. J. Schmitt, and L. E. Thode, "Numerical Simulations of Free-Electron Laser Oscillators", Nucl. Instrum. Methods Phys. Res., A285, A. Gover and V. Granatsteiı editors, pp. 186-191, North-Holland, Amsterdam,1989. 